\title{
Correction to: ADAR2 mislocalization and widespread RNA editing aberrations in C9orf72-mediated ALS/FTD
}

\author{
Stephen Moore ${ }^{1,2}$. Eric Alsop ${ }^{3} \cdot$ Ileana Lorenzini ${ }^{1} \cdot$ Alexander Starr $^{1} \cdot$ Benjamin E. Rabichow $^{1} \cdot$ Emily Mendez $^{1}$. \\ Jennifer L. Levy ${ }^{1}$. Camelia Burciu ${ }^{1} \cdot$ Rebecca Reiman $^{3}$. Jeannie Chew ${ }^{4}$. Veronique V. Belzil ${ }^{4}$. Dennis Dickson ${ }^{4}$. \\ Janice Robertson ${ }^{5} \cdot K_{i m}$ A. Staats ${ }^{6} \cdot$ Justin K. Ichida $^{6} \cdot$ Leonard Petrucelli $^{4} \cdot$ Kendall Van Keuren-Jensen $^{3} \cdot$ Rita Sattler $^{1}$
}

Published online: 26 September 2019

(c) Springer-Verlag GmbH Germany, part of Springer Nature 2019

\section{Correction to: Acta Neuropathologica (2019) 138:49-65 https://doi.org/10.1007/s00401-019-01999-w}

The original article was published erroneously without mentioning the support of the U.S. Department of Veterans Affairs Grant I01BX003625 in the funding sources or the acknowledgments section.

The corrected acknowledgements section is given below:

We would like to thank the Sattler Laboratory for suggestions and comments towards the manuscript. We would also like to thank all ALS patients and families that have contributed to this research via postmortem brain tissue donations. Specifically, we would like to thank Doug Clough for assistance with data analysis and insightful discussions. We further thank the Target ALS Human Postmortem Tissue

The original article can be found online at https://doi.org/10.1007/ s00401-019-01999-w.

Rita Sattler

Rita.Sattler@Dignityhealth.org

1 Department of Neurobiology, Barrow Neurological Institute, 355 W Thomas Road, Phoenix, AZ 85013, USA

2 School of Life Sciences, Arizona State University, Tempe, AZ, USA

3 Neurogenomics Division, Translational Genomics Research Institute, Phoenix, AZ, USA

4 Department of Neuroscience, Mayo Clinic Jacksonville, Jacksonville, FL, USA

5 Tanz Centre for Research in Neurodegenerative Diseases, University of Toronto, Toronto, Canada

6 Department of Stem Cell Biology and Regenerative Medicine, Eli and Edythe Broad Center for Regenerative Medicine and Stem Cell Biology, Keck School of Medicine, University of Southern California, Los Angeles, CA, USA
Core, New York Genome Center for Genomics of Neurodegenerative Disease, Amyotrophic Lateral Sclerosis Association and TOW Foundation for providing access to their postmortem patient tissue samples collection. We thank both the Target ALS Consortium and the New York Genome Center for access to their RNA sequencing database. In particular, we would like to thank Drs. Lyle Ostrow, Hemali Phatnani and Robert Bowser. We would also like to thank Drs. Sylvia Perez and Elliott Mufson for generously providing us with AD patient postmortem tissue samples. Further thanks go to Dr. Stella Dracheva for helpful discussions throughout this project. This work was support by the National Institute of Neurological Disorders and Stroke, NIH RO1NS085207 (RS); the Muscular Dystrophy Association (RS); the ALS Association (RS); the Robert Packard Center for ALS Research (RS), U.S. Department of Veterans Affairs grant I01BX003625 (RS); and the Barrow Neurological Foundation (RS). Part of this work was also made possible by NIH Grant R01NS097850 (JKI), US Department of Defense Grant W81XWH-15-1-0187 (JI), and grants from the Donald E. and Delia B. Baxter Foundation (JKI), the Alzheimer's Drug Discovery Foundation (JKI) and the Association for Frontotemporal Degeneration (JKI), the Harrington Discovery Institute (JKI), the Tau Consortium (JKI), the Pape Adams Foundation (JKI), the Frick Foundation for ALS Research (JKI), the Muscular Dystrophy Association (JKI), the New York Stem Cell Foundation (JKI), the USC Keck School of Medicine Regenerative Medicine Initiative (JKI), the USC Broad Innovation Award (JKI), and the Southern California Clinical and Translational Science Institute to JKI. JKI is a New York Stem Cell Foundation-Robertson Investigator and a Richard N. Merkin Scholar. We would additionally like to thank the National Institutes of Health/ National Institute of Neurological Disorders and Stroke [R35NS097273 (L.P.); P01NS084974 (L.P.); P01NS099114 (L.P.); R01NS088689 (L.P.)]; the Mayo Clinic Foundation 
(L.P.); the Amyotrophic Lateral Sclerosis Association (L.P.), the Robert Packard Center for ALS Research at Johns Hopkins (L.P.), the Target ALS Foundation (L.P.), and the James Hunter Family ALS Initiative (JR).
Publisher's Note Springer Nature remains neutral with regard to jurisdictional claims in published maps and institutional affiliations. 